\title{
An evaluation of the effectiveness of acupuncture with seven acupoint-penetrating needles on cervical spondylosis
}

\author{
This article was published in the following Dove Press journal: \\ Journal of Pain Research
}

\author{
Chun-Lei Gu' \\ Yu Yan ${ }^{2}$ \\ Ding Zhang ${ }^{3}$ \\ Ping $\mathrm{Li}^{\prime}$ \\ 'Department of Acupuncture and \\ Moxibustion, Guizhou Orthopaedics \\ Hospital, Guiyang 550007, People's \\ Republic of China; ${ }^{2}$ Department of \\ Rehabilitation, Guizhou Orthopaedics \\ Hospital, Guiyang 550007, People's \\ Republic of China; ${ }^{3}$ Department of \\ Orthopaedics, Guizhou Orthopaedics \\ Hospital, Guiyang 550007, People's \\ Republic of China
}

\begin{abstract}
Objective: To investigate the clinical efficacy of acupuncture with seven acupointpenetrating needles on patients with cervical spondylosis.

Patients and methods: Sixty patients with cervical spondylosis were grouped using computer-generated random numbers. Group A $(n=30)$ received acupuncture with seven acupoint-penetrating needles combined with traction. Group $B(n=30)$ received acupuncture of non-relevant acupuncture points combined with traction. Clinical efficacy and changes in the Visual Analogue Scale (VAS), Neck Disability Index (NDI), and Pittsburgh Sleep Quality Index (PSQI) scores in the two groups were compared.

Results: The total effective rate was significantly higher in group A $(90.0 \%)$ than in group B $(76.6 \%)(P<0.05)$. The VAS, NDI, and PSQI scores of the two groups after treatment were significantly lower than scores before treatment $(P<0.05)$. After treatment, the NDI and PSQI scores of group A were significantly lower than those in group $\mathrm{B}(P<0.05)$.

Conclusion: Acupuncture using seven acupoint-penetrating needles combined with traction was more effective, reduced neck pain, and improved sleep quality in patients with cervical spondylosis compared to acupuncture of non-relevant acupuncture points.
\end{abstract}

Keywords: cervical spondylosis, acupuncture, seven acupoint-penetrating, traction

\section{Introduction}

Cervical spondylosis is an age-related degenerative disease of the spine, primarily caused by degeneration of the cervical vertebrae, including intervertebral disc degeneration, facet joint disease, osteophyte formation, ligament thickening, and changes in cervical physiological curvature. ${ }^{1,2}$ Cervical spondylosis often occurs in middle-aged people (40-60 years old) ${ }^{3,4}$ and is a common disease in clinical orthopedics. ${ }^{5}$ In recent years, the incidence of cervical spondylosis in China is increasing, and the age of onset tends to be fairly young, with a high incidence in Chinese aged 40-50 years old. ${ }^{6}$ Cervical spondylosis is an important cause of neck pain and low back pain, and seriously affects the physical health, mental health, and life quality of patients. ${ }^{2,7}$ It is urgent to develop effective therapies for cervical spondylosis.

At present, clinical treatment of cervical spondylosis commonly includes acupuncture, massage, and traction. ${ }^{8-11}$ In this study, we evaluated the clinical efficacy of acupuncture using seven acupoint-penetrating needles on patients with cervical spondylosis. We enrolled 60 patients with cervical spondylosis and randomly
Correspondence: Chun-Lei Gu Department of Acupuncture and Moxibustion, Guizhou Orthopaedics Hospital, No. 123 Shachong South Road, Nanming District, Guiyang 550007,

People's Republic of China

Tel +8685185792149

Email chunlei_g@sina.com 
divided them into two groups: 30 patients were treated using seven acupoint-penetrating needles combined with traction, and 30 patients were treated using acupuncture of non-relevant acupuncture points combined with traction. Treatment outcomes in the two groups were compared.

\section{Patients and methods Subjects}

A total of 60 patients with cervical spondylosis who met the inclusion criteria were identified among hospitalized patients and outpatients at Guizhou Orthopaedics Hospital from September 2015 to February 2018. Patients were enrolled and randomly divided into two groups using computer-generated random numbers, with 30 patients in each group. ${ }^{12}$

\section{Inclusion criteria}

Patients included in this study (1) met the diagnostic criteria for cervical spondylosis based on clinical symptoms and neck radiography; (2) were between 18 and 70 years of age; (3) volunteered to participate in the entire treatment cycle of acupuncture and traction treatment; and (4) were of sound mind and were able to provide consent.

\section{Exclusion criteria}

Patients were excluded from this study who (1) were diagnosed with cervical cancer, tuberculosis, osteomyelitis, or severe osteoporosis; (2) had suspicious spinal injuries, including spinal cord injury; (3) were diagnosed with cervical spinal stenosis; (4) had severe heart, lung, brain, or blood system diseases; (5) had severe skin injury or skin disease at the treatment site; (6) were diagnosed with cervical spondylotic myelopathy or other types of cervical spondylosis; (7) had received cervical spine surgery or had cervical deformity; or (8) were pregnant or lactating.

\section{Treatments}

All treatments were performed with patients in a sitting position. In Group A, seven sets of acupoints were selected: Nèiguān through Dàlíng (the same meridian penetration), Băihuì through Qiándǐng (the same meridian penetration), ézhōng through Yìntáng, Năohù through Fēngfŭ (the same meridian penetration) on the left side; Fēngfŭ through Fēngchí (different meridian penetrations), WánGǔ through Yifêng (different meridian penetrations) on both sides; followed by Sānyīnjiāo through Xuánzhōng (exterior-interior meridian penetration) on the right side. Acupuncture was performed using $0.3 \mathrm{~mm} \times 40 \mathrm{~mm}$ disposable acupuncture needles (Suzhou Medical Appliance Factory). The needle was slowly inserted at an angle of $15-45^{\circ}$ in a through-acupoint direction at a depth of 0.5-2 inches for $30 \mathrm{~min}$, once per day. In addition, patients received traction using the YK-6000 warm-heat traction system (Guangzhou Yikang). Occipital-jaw belt traction was performed once a day after acupuncture at a traction angle of $5-10^{\circ}$ for $15 \mathrm{~min}$. The initial traction weight was $10 \%$ of the patient's body weight or $6 \mathrm{~kg}$. With the estimated patient tolerance as the upper limit, the weight was incrementally increased as appropriate.

In Group B, the lesion segment of cervical vertebrae and Jiájǐ on both sides of the upper and lower vertebral bodies ( 0.5 inches from the posterior median line) were selected. Acupoints were selected according to clinical symptoms: two or three acupoints among Fēngchí, Fēngfǔ, Băihuì, Shuàigǔ were selected for patients with headache and dizziness; one or two acupoints among Tīnggōng, Tīnghuì, ěrmén, Yìfēng were selected for patients with tinnitus and hearing loss; Shénmén was selected for patients with insomnia; and Nèiguān and Xīnshù were selected for patients with palpitation and arrhythmia. After routine disinfection, the disposable acupuncture needle was inserted into the acupoint quickly at a depth of $0.5-1.5$ inches, for $30 \mathrm{~min}$, once per day. The traction treatment was the same as in group A.

\section{Efficacy evaluation}

Patients received acupuncture and traction as described daily for 5 days, which constituted 1 course of treatment. Patients received 1 day of rest after each course of treatment, and treatment efficacy was evaluated after 2 courses of treatment. Treatment was deemed successful if neck and shoulder pain, finger numbness, and other clinical symptoms were removed, intervertebral foramen compression test was normal, and neck pain and insomnia scores were reduced by at least 95\%. Treatment was deemed effective if neck and shoulder pain, finger numbness, and other clinical symptoms were removed, intervertebral foramen compression test was normal, and neck pain and insomnia scores were reduced by at least $70 \%$. Treatment was deemed partially effective if neck and shoulder pain, finger numbness, and other clinical symptoms were significantly improved and neck pain and insomnia scores were reduced by at least 30\%. Otherwise, treatment was considered to have failed.

\section{Observation indicators}

The Visual Analog Scale (VAS) was used to evaluate the degree of neck pain: 0 points is painless, 1-3 points are 
classified as mild pain, 4-6 points are considered to be moderate pain, and $7-10$ points are classified as severe pain. ${ }^{13}$ The Neck Disability Index (NDI) was used to assess the activities of daily living of patients with neck problems. ${ }^{14}$ The NDI consists of 10 different functional evaluations with a total score of 50; the higher the score, the more serious the disability. The Pittsburgh Sleep Quality Index (PSQI) was used to evaluate sleep quality. Each item on this index is scored on a scale of $0-3$ points, for a total score of up to 21 points. Higher scores indicate worse sleep quality. These indicators have been shown to be applicable in Chinese populations. ${ }^{15}$

\section{Statistical analysis}

Data were expressed as mean \pm standard deviation, and compared using aStudent's $t$-test. All data were processed with SPSS 19.0 software. $P$-values less than 0.05 were considered statistically significant.

\section{Results}

As shown in Table 1, there were no significant differences in the gender, age, or duration of disease between the two groups $(P>0.05)$.

After 2 courses of treatment, the efficacy, VAS, NDI, and PSQI scores of the two groups were compared. As shown in Table 2, the total effective rate in group A was $90 \%$, compared to $76.6 \%$ in group B. The difference between the two groups was significant $(P<0.05)$.

Table I Comparison of general data of two groups of patients with cervical spondylosis

\begin{tabular}{|l|l|l|l|}
\hline Group & Gender & Age & $\begin{array}{l}\text { Duration of } \\
\text { disease }\end{array}$ \\
\cline { 2 - 4 } & Male/Female & $\begin{array}{l}\text { (years, } \\
\text { mean } \pm \text { SD) }\end{array}$ & $\begin{array}{l}\text { (month, } \\
\text { mean } \pm \text { SD) }\end{array}$ \\
\hline A & $12 / 18$ & $45.95 \pm 3.21$ & $1.82 \pm 0.75$ \\
B & $13 / 17$ & $44.62 \pm 4.34$ & $1.71 \pm 0.76$ \\
\hline
\end{tabular}

Notes: Group A received acupuncture with seven acupoint-penetrating needles combined with traction. Group B received acupuncture of non-relevant acupuncture points combined with traction.
As shown in Table 3, VAS scores of the two groups after treatment were significantly different from the scores before treatment $(P<0.05)$; but there was no significant difference between the two groups after treatment $(P>0.05)$.

As shown in Table 4, NDI scores of the two groups after treatment were significantly different from the scores before treatment $(P<0.05)$. In addition, there was a significant difference in NDI scores between the two groups after treatment $(P<0.05)$, indicating that after treatment, group A experienced significantly better NDI scores compared to group B.

As shown in Table 5, PSQI scores of the two groups after treatment were significantly different from the scores before treatment $(P<0.05)$. Moreover, there was a significant difference in PSQI scores between the two groups after treatment $(P<0.05)$, indicating that after treatment, group A experienced significantly better sleep quality compared to group B.

\section{Discussion}

According to traditional Chinese medicine, the occurrence and development of cervical spondylosis is closely related to dysfunction of the liver, spleen, and kidney. The concept of yin and yang is fundamental in traditional Chinese medicine. In acupuncture, yang meridians all pass through the neck, the Du meridian and the bladder meridian of the foot-Taiyang pass through Hòuxiàng, the triple-warmer channel of the hand-Shaoyang and the gallbladder meridian of the footShaoyang pass through the throat, the large intestine meridian of the hand-Yangming and the stomach meridian of the foot-Yangming pass through the front side of the neck, while the Ren meridian is in the middle of the anterior neck. Because all yang meridians start and end in the head, the head is the place where yang meridians gather. Among all yang meridians, the bladder meridian, the gallbladder meridian, and the Du meridian have the greatest influence on the neck. Therefore, in this study we selected the acupoints of the Du meridian, bladder meridian, and gallbladder meridian at the neck to be penetrated by the seven acupoint-penetrating needles, transfusing the meridians and collaterals to treat the symptoms; then we selected the acupoints of the liver, kidney, spleen, and heart meridians for penetration, adjusting

Table 2 Comparison of clinical efficacy between two groups

\begin{tabular}{|l|l|l|l|l|l|l|}
\hline Group & Cases & Cure & Effectiveness & Partial effectiveness & Failure & Effective rate \\
\hline A & 30 & 10 & 12 & 5 & 3 & $90.0 \%$ \\
B & 30 & 5 & 9 & 9 & 7 & $76.7 \% *$ \\
\hline
\end{tabular}

Notes: ${ }^{*} P<0.05$ compared with group B. Effective rate was calculated as a case without failure/total case. Group A received acupuncture with seven acupointpenetrating needles combined with traction. Group B received acupuncture of non-relevant acupuncture points combined with traction. 
Table 3 Comparison of VAS scores in 2 groups before and after treatment

\begin{tabular}{|l|l|l|l|}
\hline \multirow{2}{*}{ Group } & \multirow{2}{*}{$\mathbf{l}$} & \multicolumn{2}{|l|}{ VAS score (mean \pm SD) } \\
\cline { 3 - 4 } & & Before treatment & After treatment \\
\hline A & 30 & $5.63 \pm 1.03$ & $2.39 \pm 0.35^{*}, \#$ \\
B & 30 & $5.78 \pm 0.89$ & $2.88 \pm 0.60^{*}$ \\
\hline
\end{tabular}

Notes: $* P<0.05$ compared with before treatment; ${ }^{\#} P>0.05$ compared with group $B$ after treatment. Group A received acupuncture with seven acupoint-penetrating needles combined with traction. Group B received acupuncture of non-relevant acupuncture points combined with traction.

Abbreviation: VAS, Visual Analogue Scale.

Table 4 Comparison of NDI scores in 2 groups before and after treatment

\begin{tabular}{|l|l|l|l|}
\hline Group & $\mathbf{n}$ & \multicolumn{2}{|l|}{ NDI score (mean \pm SD) } \\
& & Before treatment & After treatment \\
\hline A & 30 & $24.90 \pm 3.81$ & $6.43 \pm 3.70^{*}, \#$ \\
B & 30 & $23.16 \pm 2.90$ & $10.75 \pm 3.68^{*}$ \\
\hline
\end{tabular}

Notes: $* P<0.05$ compared with before treatment; ${ }^{\#} P<0.05$ compared with group $B$ after treatment. Group A received acupuncture with seven acupoint-penetrating needles combined with traction. Group B received acupuncture of non-relevant acupuncture points combined with traction.

Abbreviation: NDI, Neck Disability Index.

Table 5 Comparison of PSQI scores in 2 groups before and after treatment

\begin{tabular}{|l|l|l|l|}
\hline Group & $\mathbf{n}$ & \multicolumn{2}{|l|}{ PSQI score (mean \pm SD) } \\
& & Before treatment & After treatment \\
\hline A & 30 & $17.90 \pm 3.81$ & $5.43 \pm 3.70^{*}, \#$ \\
B & 30 & $17.16 \pm 2.90$ & $9.24 \pm 3.68^{*}$ \\
\hline
\end{tabular}

Notes: ${ }^{* P}<0.05$ compared with before treatment; ${ }^{\#} P<0.05$ compared with group $B$ after treatment. Group A received acupuncture with seven acupoint-penetrating needles combined with traction. Group B received acupuncture of non-relevant acupuncture points combined with traction.

Abbreviation: PSQI, Pittsburgh Sleep Quality Index.

viscera function as appropriate. Selecting acupoints on only one side of the limbs can reduce the discomfort caused by acupuncture, and the meridian penetration method used in this study can promote the movement of yang meridians and reduce pain.

The efficacy observed with seven acupoint-penetrating needles combined with traction to treat cervical spondylosis is due to several factors. First, the selected acupoints, such as Năohù, Fēngfũ, Fēngchí, WánGǔ, and Yìfēng, are mostly distributed at the junctions of tendons, ligaments, and bones. These points are the stress concentration points of muscle fiber bundles or tendons, and are adjacent to important muscles and nerve tissues in the neck. Point-through-point acupuncture of these acupoints can improve the high tension state of spasm or contracture of soft tissues such as cervical fascia, muscles, ligaments, and articular capsules, reduce the bad traction of these tissues on the cervical vertebral body, nerves, and blood vessels, and correct the state of cervical instability, alleviating pain and improving cervical dysfunction. Second, acupoint penetration at the head can activate the reticular activation system in the brain, increase the excitability of the cerebral cortex, improve local blood circulation of the brain, and accelerate metabolism, thereby improving patients' sleep quality, anxiety, general discomfort, and other symptoms. Third, intermittent traction in a sitting position can effectively reduce the spasticity of muscles and ligaments around the cervical vertebrae, relieve the pressure on the vertical axis of the cervical vertebrae, increase intervertebral space and intervertebral foramen, relieve compression of the nerve roots by osteophytes or herniated intervertebral discs, and reduce pressure in intervertebral discs.

Our study has some limitations. Patients had experienced cervical spondylosis for generally short periods of time, and spontaneous remission is possible. In addition, the placebo effect could play a role in treatment outcomes. Due to patient preferences we did not enroll a control group without any treatment, and group B is not a perfect control group. All three scores being evaluated are subjective, and large sample sizes can help to verify the effectiveness of the proposed treatment.

\section{Conclusion}

Our results showed that acupuncture using seven acupointpenetrating needles combined with traction was more effective, reduced neck pain, and improved sleep quality in patients with cervical spondylosis.

\section{Ethics approval and informed consent}

This study was approved by the ethics committee of Guizhou Orthopaedics Hospital and conducted in accordance with the Declaration of Helsinki. Written informed consent was obtained from all subjects.

\section{Disclosure}

The authors report no conflicts of interest in this work.

\section{References}

1. McDonnell M, Lucas P. Cervical spondylosis, stenosis, and rheumatoid arthritis. Med Health R I. 2012;95(4):105-109. 
2. Peng B, DePalma MJ. Cervical disc degeneration and neck pain J Pain Res. 2018;11:2853-2857.

3. Emery SE. Cervical spondylotic myelopathy: diagnosis and treatment. J Am Acad Orthop Surg. 2001;9(6):376-388.

4. Lee MJ, Cassinelli EH, Riew KD. Prevalence of cervical spine stenosis. Anatomic study in cadavers. J Bone Joint Surg Am. 2007;89(2):376-380

5. Tracy JA, Bartleson JD. Cervical spondylotic myelopathy. Neurologist. 2010;16(3):176-187.

6. Bai J, Yu K, Sun Y, Kong L, Shen Y. Prevalence of and risk factors for Modic change in patients with symptomatic cervical spondylosis: an observational study. J Pain Res. 2018;11:355-360.

7. Elder BD, Witham TF. Low Back Pain and Spondylosis. Semin Neurol. 2016;36(5):456-461.

8. Nakajima M, Inoue M, Itoi M, Kitakoji H. Clinical effect of acupuncture on cervical spondylotic radiculopathy: results of a case series. Acupunct Med. 2013;31(4):364-367.

9. Kong LJ, Zhan HS, Cheng YW, Yuan WA, Chen B, Fang M. Massage therapy for neck and shoulder pain: a systematic review and meta-analysis. Evid Based Complement Alternat Med. 2013;2013:1-10.

10. Sun ZR, Yue JH, Zhang QH. Electroacupuncture at Jing-jiaji points for neck pain caused by cervical spondylosis: a study protocol for a randomized controlled pilot trial. Trials. 2013;14:360.
11. Que Q, Ye X, Su Q, et al. Effectiveness of acupuncture intervention for neck pain caused by cervical spondylosis: study protocol for a randomized controlled trial. Trials. 2013;14:186.

12. Kobayashi A, Suginuma K, Furuichi M. TRISim: a triage simulation system to exploit and assess triage operations for hospital managers Development, validation and experiment. Comp Model Eng Sci. 2017;113:117-149.

13. Martins PC, Couto TE, Gama AC. Auditory-perceptual evaluation of the degree of vocal deviation: correlation between the visual analogue scale and numerical scale. Codas. 2015;27 (3):279-284.

14. Sundseth J, Kolstad F, Johnsen LG, et al. The Neck Disability Index (NDI) and its correlation with quality of life and mental health measures among patients with single-level cervical disc disease scheduled for surgery. Acta Neurochir. 2015;157 (10):1807-1812.

15. Zhou XY, Xu XM, Fan JP, et al. Cross-cultural validation of simplified Chinese version of spine functional index. Health Qual Life Outcomes. 2017;15(1):203.

\section{Publish your work in this journal}

The Journal of Pain Research is an international, peer reviewed, open access, online journal that welcomes laboratory and clinical findings in the fields of pain research and the prevention and management of pain Original research, reviews, symposium reports, hypothesis formation and commentaries are all considered for publication. The manuscript management system is completely online and includes a very quick and fair peer-review system, which is all easy to use. Visit http:// www.dovepress.com/testimonials.php to read real quotes from published authors. 